\title{
IN VIVO PROTON MR SPECTROSCOPY IN DIAGNOSIS OF INTRACRANIAL CYSTIC LESIONS
}

\author{
Sukumar Ramasamy11, Periakaruppan Alagappan², Amarnath Chellathurai3, Priya Muthaiyan4, Suja Rajan ${ }^{5}$
}

1 Professor, Department of Radiodiagnosis, Stanley Medical College, Chennai, Tamilnadu.

${ }^{2}$ Associate Professor, Department of Radiodiagnosis, Stanley Medical College, Chennai, Tamilnadu.

3 Professor, Department of Radiodiagnosis, Stanley Medical College, Chennai, Tamilnadu.

${ }^{4}$ Assistant Professor, Department of Radiodiagnosis, Stanley Medical College, Chennai, Tamilnadu.

5Junior Resident, Department of Radiodiagnosis, Stanley Medical College, Chennai, Tamilnadu.

\section{BACKGROUND}

ABSTRACT

Magnetic resonance spectroscopy (MRS) ${ }^{1}$ is a non-invasive diagnostic tool that has been used to detect biochemical environment and the concentration of the metabolites in various tissues, which correlate with physiologic and pathological processes.

Aims and Objectives: To determine the use of proton in vivo spectroscopy in diagnosing intracranial cystic lesions by providing additional metabolic information.

\section{MATERIALS AND METHODS}

This is a retrospective, descriptive, single institutional study with study population of 108 patients with intracranial cystic lesions. 1.5 Tesla Siemens system was used to perform MRI brain conventional sequences along with MR spectroscopy.

\section{RESULTS}

The pyogenic abscess showed amino acids, alanine, lactate, lipid and acetate peaks. Tuberculous abscess showed tall lipid with lactate peak and absent amino acids. Both neurocysticercosis and hydatid cysts showed succinate and lactate peaks with minimal acetate along with creatinine peak in NCC. Lactate was found in the benign non-neoplastic cysts studied including arachnoid cysts, porencephalic cysts, epidermoid cysts, craniopharyngioma and DNET. While choroid fissural cyst, choroid plexus cysts, ganglioglioma showed no specific metabolite. One colloid cyst studied showed increased NAA peak at 2.0 ppm. Among the malignant lesions, high-grade tumours showed lipid peak in addition to increased choline, lactate peaks, decreased creatinine and NAA peak. Metastatic lesions showed lipid and lactate peaks.

\section{CONCLUSION}

Magnetic resonance spectroscopy has a definitive complementary role in aiding the diagnosis of various intracranial cystic lesions.

\section{KEY WORDS}

Magnetic Resonance Spectroscopy, Pyogenic Abscess, Epidermoid, Neuroglial Cyst, Neurocysticercosis, Hydatid Cyst.

HOW TO CITE THIS ARTICLE: Ramasamy S, Alagappan P, Chellathurai A, et al. In vivo proton MR spectroscopy in diagnosis of intracranial cystic lesions. J. Evolution Med. Dent. Sci. 2018;7(19):2398-2403, DOI: 10.14260/jemds/2018/540

\section{BACKGROUND}

Magnetic resonance spectroscopy (MRS) ${ }^{1}$ is a non-invasive diagnostic tool that has been used to detect biochemical environment and the concentration of the metabolites in various tissues, which correlate with physiologic and pathological processes. Proton MRS is widely used for brain pathologies including tumours, strokes, seizure disorders, hypoxia, white matter diseases, stroke, multiple sclerosis, Alzheimer's disease, depression, etc. In Brain MR spectroscopy, spectrum ${ }^{2}$ obtained shows the metabolites at specific ppm.

A variety of intracranial lesions including gliomas, metastases, craniopharyngiomas, abscesses, parasitic cysts (such as cysticercosis), epidermoids and arachnoid cysts may appear as mainly cystic masses on magnetic resonance (MR) and computed tomography (CT) studies of the brain.

'Financial or Other Competing Interest': None.

Submission 22-03-2018, Peer Review 23-04-2018,

Acceptance 30-04-2018, Published 07-05-2018.

Corresponding Author:

Dr. Periakaruppan Alagappan,

Department of Radiodiagnosis,

Stanley Medical College, Royapuram,

Chennai-600001, Tamilnadu.

E-mail: smallarunrad@yahoo.co.in

DOI: $10.14260 /$ jemds $/ 2018 / 540$
The combined CT and MRI findings along with clinical information do not always characterise the lesions. MR spectroscopy may provide additional information in such cases.

Brain abscesses are localised pyogenic infection, which may mimic cystic tumours. Neurocysticercosis is intracranial parasitic infection by Taenia solium, which present as multiple lesions typically in the gray matter-white matter junction and deep sulci. Tuberculous abscess may be a focal manifestation, imaging features vary depending on the stage. Cerebral hydatid cysts (HC) are extremely rare, usually seen as unilocular cyst with ring enhancement which rarely calcifies.

Arachnoid cysts are CSF containing cysts lined by a layer of flattened mature arachnoid cells caused by maldevelopment of meninges. Choroid fissural cyst are shallow cysts noted in the fissure caused by maldevelopment of embryonic tela choroidea. Ependymal cysts are common benign cysts noted in the atrium of lateral ventricle. Porencephaly is congenital or acquired CSF filled cavities due to destructive process such as intraparenchymal haemorrhage, infection or surgery. The overall prevalence of porencephaly is $2.5 \%$ of congenital lesions in children. In MRI these are CSF dense lesion, which do not enhance or show diffusion restriction. 
Choroid plexus cysts are non-neoplastic, noninflammatory cysts commonly located within the body of the plexus. These cystic lesions may be associated with aneuploidy, particularly trisomy 18 . Neurenteric cysts are rare, benign, congenital endodermal lesions, more commonly located in the spine than in the brain (posterior fossa). Colloid cysts are endodermal derivative cysts, $50 \%$ of colloid cysts are hyperintense on T1-weighted images, hypointense on $\mathrm{T} 2 \mathrm{w}$ images. Their rupture may lead to chemical meningitis.

Epidermoid cysts represent ectopic epidermal rest due to defect in neural tube closure during gastrulation. T2 bright lesions are partly suppressed by FLAIR, shows restricted diffusion which is diagnostic. DNET are low grade, cortical based mixed neuronal-glial tumour may be associated with cortical dysplasia. Ganglioglioma are well-differentiated neuroepithelial tumours with varying amounts of solid and cystic components. DNET and Ganglioglioma both are typically seen in temporal lobes presenting with seizures.

Here, we are assessing the usefulness of proton in vivo spectroscopy in providing additional information regarding various intracranial cystic lesions and differentiating them as infectious, congenital, benign and malignant lesions.

\section{MATERIALS AND METHODS}

This is a retrospective, descriptive, single institutional study conducted during the time period of December 2015 December 2017. The selected population consists of 108 patients ( 56 males and 52 females).

\section{Inclusion Criteria}

Patients with intracranial cystic lesions, either incidentally or intentionally who came to our department for MRI brain study. Informed consent was obtained from the patients. In case of multiple lesions, lesion with largest cystic component was selected. Exclusion criteria- Lesions below the size of 5 mm were not taken up for study. Lesions very close to scalp were excluded due to contamination of value by scalp fat. Postoperative patients and post radiation were excluded from the study.

All participants were studied with a 1.5-T whole-body MR imager (Siemens Magnetom Aera, Germany) equipped with high-performance gradients using a manufacturer-supplied head coil. Routine sequences performed in all patients were sagittal T1-weighted (608/17/1 [TR/TE/excitations]), axial fast spin-echo T2-weighted (4450/98/1), coronal fast fluidattenuated inversion recovery (FLAIR) (90000/90/1, TI 2.2 seconds), diffusion-weighted sequences and ADC. In general, all axial sequences used 5-mm thickness with intersection gap of $3 \mathrm{~mm}$, a $305 \times 448$ matrix, the same imaging angle along the orbitomeatal line and a $200 \mathrm{~mm}$ field of view.

In all patients, proton MR spectroscopy was performed by using chemical shift imaging (CSI) sequence (2000/144 and 135/35 [TR/TE]), FOV $160 \times 160 \mathrm{~mm}$, sample volume size 27 x 27 x $20 \mathrm{~mm}$; matrix size- 128 x $128 \mathrm{~mm}$; section thickness$5 \mathrm{~mm}$. Multivoxel grid was placed in cyst or cystic component in case of necrotic lesions.

Care was taken not to include bony regions or haemorrhagic portions to exclude contamination of spectroscopic values. Typical acquisition time per spectral acquisition was $3 \mathrm{mts} 10 \mathrm{~s}$. The spectra were assessed independently by two experienced Radiologists. Concordance between their findings was taken as a positive indicator that the spectrum was diagnostic for the pathology under consideration.

Following that based on the MR findings few patients were further subjected to IV contrast of gadolinium at a dose of $0.1 \mathrm{mmol} / \mathrm{kg}$.

The diagnosis of brain abscess was confirmed by either microscopic examination of aspirated fluid or histopathological examination of surgically excised material. Histologic diagnosis was confirmed in all patients with epidermoid cysts, tumours and metastases from specimen obtained by surgical resection. The diagnosis of benign nonsurgical cyst was confirmed by imaging follow-up after 6 months while neurocysticercosis, hydatid cysts and tuberculous abscesses by post-treatment follow-up after 6 months.

\section{RESULTS}

The abscesses $(n=5)$ showed amino acid (valine, leucine, isoleucine), lactate, lipid, acetate and alanine peaks. All 14 neurocysticercosis cases showed lactate, succinate and creatinine peaks. Of the 4 tuberculous abscesses all showed lipid peak, while only 2 of them showed lactate peak. The two hydatid cysts showed lipid, lactate, succinate and alanine peak.

Benign non-neoplastic cysts studied including 21 arachnoid cysts, 2 choroid fissural cyst and 2 choroid plexus showed non-specific signals. 12 porencephalic cysts studied showed mild lactate peaks and no other specific peak. 6 epidermoid cysts were studied- all showed mild lactate peak and no other specific peaks. Cystic component in 2 craniopharyngiomas showed lactate peak. Among 6 DNETS studied, two showed minimal lactate peaks and four others showed no specific signals. The 2 ganglioglioma studied minimal lactate with absent $\mathrm{Cr}$, Choline peaks. One colloid cyst studied showed NAA peak at $2.0 \mathrm{ppm}$.

Among the 29 neoplastic lesions with necrotic components, 13 low-grade tumours all showed increased choline peak, decreased creatinine, NAA peak along with lactate peak. The 10 high-grade neoplasms including gliomas, medulloblastoma showed increased choline, decreased creatinine, NAA peaks along with lipid and lactate peaks. The 6 cystic metastatic lesions showed lipid, lactate peaks.

\section{RESULTS}

\begin{tabular}{|c|c|}
\hline Age & Number of Patients \\
\hline$<5$ & 7 \\
\hline $5-10$ & 15 \\
\hline $11-20$ & 19 \\
\hline $21-30$ & 11 \\
\hline $31-40$ & 13 \\
\hline $41-50$ & 17 \\
\hline $51-60$ & 8 \\
\hline $61-70$ & 12 \\
\hline$>71$ & 6 \\
\hline \multicolumn{2}{|c|}{ Table 1a. Age Distribution } \\
\hline
\end{tabular}




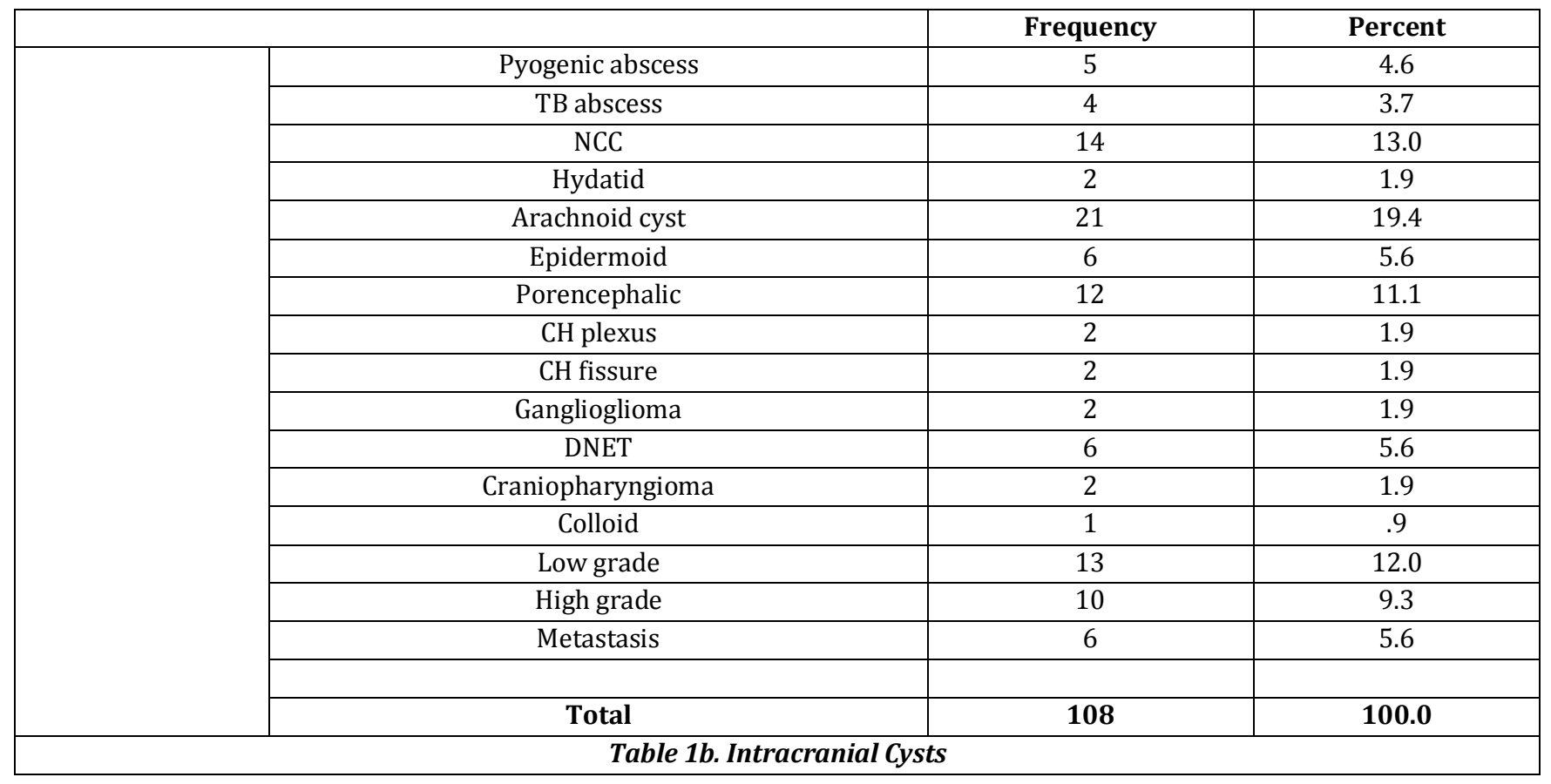

\begin{tabular}{|c|c|c|}
\hline CYST & $\begin{array}{c}\text { Total } \\
\text { No. }\end{array}$ & MRS Findings \\
\hline Brain abscess & 5 & $\begin{array}{c}\text { Lactate- } 5(100 \%) \\
\text { Branched chain Amino acids- } 5(100 \%) \\
\text { Alanine- } 5(100 \%) \\
\text { Acetate- } 5(100 \%) \\
\text { Succinate- } 1(20 \%)\end{array}$ \\
\hline Tuberculous abscess & 4 & $\begin{array}{c}\text { Lipid- } 4(100 \%) \\
\text { Lactate- } 2(50 \%)\end{array}$ \\
\hline Neurocysticercosis & 14 & $\begin{array}{c}\text { Lactate- } 14(100 \%) \\
\text { Succinate- } 14(100 \%) \\
\text { Choline- } 14(100 \%) \\
\text { Acetate- } 3(21.4 \%) \\
\text { NAA, Cr- } 9(64.3 \%)\end{array}$ \\
\hline Hydatid cyst & 2 & $\begin{array}{c}\text { Lactate- } 2 \text { (100\%), Alanine- } 1(50 \%) \\
\text { Succinate- } 2(100 \%) \text {, Acetate- } 1(50 \%)\end{array}$ \\
\hline Arachnoid cyst & 21 & $\begin{array}{c}\text { Lactate- } 16(76.2 \%) \\
\text { No specific metabolites- } 5\end{array}$ \\
\hline Porencephalic cyst & 12 & Lactate- $12(100 \%)$ \\
\hline Epidermoid cyst & 6 & Lactate- $6(100 \%)$ \\
\hline Choroid fissural cyst & 2 & No specific metabolites \\
\hline Choroid plexus cyst & 2 & No specific metabolites \\
\hline Ganglioglioma & 2 & No specific metabolites \\
\hline DNET & 6 & $\begin{array}{c}\text { Lactate- } 2(33.3 \%) \\
\text { No specific metabolites- } 4\end{array}$ \\
\hline Craniopharyngioma & 2 & Lactate- $2(100 \%)$ \\
\hline Colloid cyst & 1 & NAA- $1(100 \%)$ \\
\hline Low-grade glioma & 13 & $\begin{array}{c}\text { Increased Choline- } 13(100 \%) \\
\text { Reduced NAA, Creatinine- } 13(100 \%) \\
\text { Lactate- } 13(100 \%) \\
\text { Lipid- } 2(15.4 \%)\end{array}$ \\
\hline High-grade glioma & 10 & $\begin{array}{c}\text { Increased Choline- } 10(100 \%) \\
\text { Reduced NAA, Creatinine- } 10(100 \%) \\
\text { Lactate- } 10(100 \%) \\
\text { Lipid- } 10(100 \%)\end{array}$ \\
\hline Metastasis & 6 & $\begin{array}{l}\text { Lactate- } 6 \text { (100\%), Lipid- } 6 \text { (100\%) } \\
\text { Increased Choline- } 2 \text { (33.3\%) }\end{array}$ \\
\hline Table 2 & in var & sed in the Table Below \\
\hline
\end{tabular}




\begin{tabular}{|c|c|c|}
\hline Metabolite Peak & Number & $\begin{array}{c}\text { Lesions } \\
\end{array}$ \\
\hline Lactate & $90(83 \%)$ & $\begin{array}{c}\text { Pyogenic abscess- } 5(100 \%) \\
\text { Tuberculous abscess- } 2(50 \%) \\
\text { Neurocysticercosis- } 14(100 \%) \\
\text { Hydatid cyst- } 2(100 \%) \\
\text { Arachnoid cyst- } 16(63.8 \%) \\
\text { Porencephalic cyst- } 12(100 \%) \\
\text { Epidermoid cyst- } 6(100 \%) \\
\text { DNET- } 2(33.3 \%) \\
\text { Low-grade glioma- } 13(100 \%) \\
\text { High-grade glioma- } 10(100 \%) \\
\text { Metastasis- } 6(100 \%)\end{array}$ \\
\hline Lipid & $22(20.4 \%)$ & $\begin{array}{c}\text { Tuberculous abscess- } 4(100 \%) \\
\text { High-grade glioma- } 10(100 \%) \\
\text { Low-grade glioma- } 2(15.4 \%) \\
\text { Metastasis- } 6(100 \%) \\
\end{array}$ \\
\hline Branched amino acids & $5(4.6 \%)$ & Pyogenic abscess- 5 (100\%) \\
\hline Succinate & $17(15.7 \%)$ & $\begin{array}{c}\text { Pyogenic abscess- } 1(20 \%) \\
\text { Neurocysticercosis- } 14(100 \%) \\
\text { Hydatid cyst- } 2(100 \%)\end{array}$ \\
\hline Acetate & $9(8.3 \%)$ & $\begin{array}{c}\text { Pyogenic abscess- } 5(100 \%) \\
\text { Neurocysticercosis- } 3(21.4 \%) \\
\text { Hydatid cyst- } 1(50 \%) \\
\end{array}$ \\
\hline Choline & $34(31.5 \%)$ & $\begin{array}{c}\text { Low-grade glioma- } 13(100 \%) \\
\text { High-grade glioma- } 10(100 \%) \\
\text { Metastasis- } 2(33.3 \%) \\
\text { NCC- } 9(64.3 \%)\end{array}$ \\
\hline Creatinine & $14(100 \%)$ & Neurocysticercosis- 14 (100\%) \\
\hline NAA & $10(9.3 \%)$ & $\begin{array}{c}\text { Colloid cyst- } 1(100 \%) \\
\text { Neurocysticercosis }(64.3 \%)\end{array}$ \\
\hline Alanine & $13(12 \%)$ & $\begin{array}{c}\text { Pyogenic amino acids- } 5(100 \%) \\
\text { Neurocysticercosis- } 7(50 \%) \\
\text { Hydatid cyst- } 1(50 \%)\end{array}$ \\
\hline
\end{tabular}

\begin{tabular}{|c|c|c|c|c|c|c|c|c|c|c|c|c|c|c|c|}
\hline DIAGNOSIS & Cho* $^{*}$ & $\mathrm{Cr}$ & NAA & Suc & Acet & Alan & $\begin{array}{c}\text { AA Val, } \\
\text { Leu, Ileu }\end{array}$ & Gly & Lactate & Lipid & & Lip & AA & Ur & BB \\
\hline Brain abscess & - & - & - & $+/-$ & + & + & + & - & ++ & - & & & $\uparrow$ & & \\
\hline Neurocysticercosis & $+/-$ & + & $+/-$ & ++ & $+/-$ & + & - & - & +++ & - & & & & & \\
\hline Tuberculoma & - & - & - & - & - & - & - & - & $+/-$ & +++ & + & & & & \\
\hline Hydatid & - & - & - & +++ & $+/-$ & $+/-$ & - & - & ++ & - & & & & & \\
\hline $\begin{array}{c}\text { Arachnoid cyst, } \\
\text { Choroid plexus cyst, } \\
\text { Choroid fissural cyst, }\end{array}$ & - & - & - & - & - & - & - & - & $+/-$ & - & & & & & \\
\hline Epidermoid & - & - & - & - & - & - & - & - & + & - & & & & $\uparrow$ & \\
\hline Porencephalic cyst & - & - & - & - & - & - & - & - & + & - & & $=-$ & & & $\uparrow \uparrow$ \\
\hline Colloid cyst & - & - & + & - & - & - & - & - & - & - & & $\uparrow$ & & & \\
\hline Ganglioglioma & - & - & - & - & - & - & - & - & $+/-$ & - & & & & & \\
\hline DNET & - & - & - & - & - & - & - & - & $+/-$ & - & & & & & \\
\hline Low-grade tumours & $\uparrow$ & $\downarrow$ & $\downarrow$ & - & - & - & - & - & + & - & & & & & \\
\hline High-grade tumours & $\uparrow$ & $\downarrow$ & $\downarrow$ & - & - & - & - & - & + & + & & & & & \\
\hline Metastasis & - & - & - & - & - & - & - & - & + & + & & & & & \\
\hline DNET & $=$ & $=$ & $=$ & & & & & + & & & & & & & \\
\hline Neuroglial cysts & - & - & + & & & & & - & & & & & & & \\
\hline
\end{tabular}

Cho- choline, Cr- creatinine, NAA- Acetylaspartate, Sucsuccinate, Acet- Acetate, Ala- Alanine, AA- Aminoacids, GlyGlycine

\section{DISCUSSION}

The MRI images and spectroscopic patterns of the 108 patients with intracranial cystic lesions included in our study were analysed and spectral peaks of specific metabolites were identified.
All the pyogenic abscesses in our study showed cytosolic branched chain amino acid, lactate, alanine, acetate and one of them showed succinate peak. The amino acids were explained by the presence of large amounts of neutrophils and pus, which on cell lysis secrete proteolytic enzymes, which result in the amino acid content. In the previous studies by RK Gupta ${ }^{2}$ et al, pyogenic abscesses showed amino acid, lactate and alanine peaks. Similarly, in study by Luthra ${ }^{3}$ et al, pyogenic abscesses showed amino acid, lactate, lipid, 
acetate and succinate peaks. While Hyun Chang4 et al, demonstrated amino acids, lactate, acetate and succinate peaks. Poptani ${ }^{5}$ et al demonstrated amino acids, lactate and acetate peaks in the pyogenic abscesses.

In study conducted by Lai $\mathrm{PH}^{6}$ et al, he differentiated aetiology of the pyogenic abscess by MR spectroscopy by absence of succinate peaks in aerobes and facultative aerobic bacteria. In case of aerobic metabolism, pyruvate from glycolysis may enter into the citric acid cycle and in facultative anaerobes in the presence of oxygen. Thus, although succinate is one of the intermediate metabolites of the citric acid cycle, succinate does not accumulate and gets transformed into the next intermediate form. This event results in the absence of succinate in these abscesses. Monika Garg7 et al, RK Gupta ${ }^{2}$ et al also differentiated aerobes showed amino acids, lactate, lipid and alanine. Glycine from anaerobes showed acetate, minimal succinate peaks in addition to it.

In contrast to pyogenic abscesses, tubercular abscesses all showed tall lipid peak, while only 2 of them showed lactate peak. There was no evidence of amino acid, acetate, succinate, alanine peaks in contrast to pyogenic abscess. This is in accordance with previous studies conducted by Luthra ${ }^{4}$ et al, RK Gupta ${ }^{2}$ et al. The presence of lipid peak can be explained by the lipid rich mycolic acid present in the tuberculous cell wall.

The neurocysticercosis cysts we studied all showed lactate, succinate and creatinine peaks, while 3 showed very minimal acetate peaks. 7 of them showed alanine peaks. In previous study by Rakesh Gupta ${ }^{2}$ et al, neurocysticercosis cysts showed resonances in lactate, cytosolic amino acids, succinate, alanine with minimal acetate showing acetate: succinate ratio $<1$ along with NAA, choline and creatinine. This ratio will be seen reversed in the case of pyogenic abscesses. In our study very minimal acetate was visible in 3 of them, while all the cysts showed significant succinate peaks. AT $1.3 \mathrm{ppm}$, there was significant overlap between lactate, amino acids and alanine signals. The individual signals could not be differentiated clearly.

All neurocysticercosis cysts showed choline peak, while 9 of them showed NAA, creatinine peaks. The presence of NAA, choline and creatinine may be attributed to the solid parenchymal tissue contaminating the voxel. This contamination interfered with creatinine peak, which is the differentiating feature of large neurocysticercosis cyst from hydatid cyst. According to previous literature, Chang et al, ${ }^{4}$ Creatinine is seen in viable cysts because of the muscle fibres noted in the bladder and scolex of the cyst. The creatinine peak can be explained by diffusion of creatinine molecule into cysts from surrounding parenchyma and creatinine present in the bladder wall of cysticercosis.

The hydatid cysts we studied showed lactate and tall succinate peak. One of them showed alanine and minimal acetate. Creatinine and Cytosolic amino acids were not detectable and acetate: succinate ratio was less than 1 . In a study by Monika ${ }^{7}$ et al and Garg8 et al stated that there was absence of creatinine in hydatid cyst in contrast to neurocysticercosis cyst. Further, they postulated that fertile hydatid cysts show presence of malate and fumarate. Gupta ${ }^{2}$ et al demonstrated similar findings and he also demonstrated malate peak at $4.3 \mathrm{ppm}$ and fumarate peak at $6.5 \mathrm{ppm}$ in fertile hydatid cysts. However, this finding was not demonstrated in our study.

According to Gupta $\mathrm{K}^{2}$ et al, succinate and acetate can be found in both parasitic pyogenic abscesses, while in pyogenic abscesses showed acetate: succinate $>1$ and in parasitic abscesses ratio is reversed. Succinate is more specific in parasite, preferentially in cestodes.

The epidermoid cysts we included in our study showed lactate peak. Poptani ${ }^{5}$ et al and Lal et al $^{9}$ showed lactate peak consistent in all epidermoid cysts. In case of arachnoid cysts, Lai $^{9}$ et al showed minimal lactate peak, while study conducted by Bhuyan ${ }^{9}$ et al and Chang et $\mathrm{al}^{4}$ showed no specific metabolite peak. In the 21 arachnoid cysts we studied, we found 16 cysts with lactate peaks, while others showed no specific metabolite peak in the spectroscopy. Similarly, few DNETS and 2 craniopharyngiomas showed minimal lactate peak in the cystic component of the lesion.

In study conducted by Hyun Chang ${ }^{4}$ et al, porencephalic cysts showed no specific metabolite peak. In our study, MR spectroscopy shows varying levels of lactate in the porencephalic cysts. Other non-specific cysts like choroid fissural cyst, choroid plexus and ganglioglioma showed no specific metabolite peak.

According to Hingwala ${ }^{10}$ and Periakaruppan ${ }^{11}$ et al, a large peak was found at $2.0 \mathrm{ppm}$ is found in the place of NAA in neurenteric cysts which was not present in other benign or developmental cysts. One neurenteric cyst we studied showed rise in NAA peak with no other significant finding.

Total of 29 neoplastic lesions are included in our study. Among them, 13 low-grade tumours showed reduced NAA, increased choline and lactate peaks with only 2 showing lipid peak. 10 cases of high-grade glioma showed increased choline peak, reduced NAA, $\mathrm{Cr}$ along with lipid and lactate component. In previous studies like Lai et al, Chang ${ }^{4}$ et al and Rakesh Gupta ${ }^{2}$ et al presence of lactate/ lipids (1.33 ppm) and elevated choline (3.2 ppm) signals suggest malignant tumours. In most gliomas and metastases, only a lactate resonance was observed with a higher lactate peak along with lipids in high-grade gliomas and metastasis. In the 10 high-grade gliomas we studied including 2 medulloblastomas, all of them showed similar findings.

According to Poptani ${ }^{5}$ et al, cystic metastasis lesions showed lipid, lactate peaks with absence of NAA, CholineCreatinine peaks. This is also observed in the 6 cystic metastatic lesions taken up in our study. 2 of the metastases also showed moderate increase in choline peak.

Lactate was the most common metabolite found in these cysts- 90 in 108 cysts included in our study rendering it as non-specific finding.

In concluding our study many of the cysts like arachnoid cyst, colloid cyst and porencephalic cyst can be diagnosed by imaging appearance alone. Similarly, the diagnosis of infective abscesses and epidermoid cysts can be clinched by diffusion restriction. This study provides additional information in differentiating pyogenic abscess from tuberculous abscess by presence of aminoacids in pyogenic abscess and tall lipid peak in tuberculous abscess. Further, it gives a clue about the aetiologic agent of the pyogenic abscesses, either aerobic or anaerobic agent by the absence of succinate peak in aerobic bacteria. Another significant advantage of this study is differentiating large 
neurocysticercosis cyst from other parasitic cysts like hydatid cyst by presence of creatinine. The presence of NAA peak at $2.02 \mathrm{ppm}$ is an unique finding of neuroglial cyst and differentiates it from other benign cysts like arachnoid cyst and porencephalic cyst. The presence of lipid in addition to lactate, choline peak and reduced NAA and creatinine indicates an aggressive high-grade malignant lesion.

Limitation in our study includes small sample size of cyst including hydatid and neuroglial cyst. This needs to be studied further with a larger number of sample. Around nine of the neurocysticercosis cysts showed NAA, Choline, Creatinine peaks likely due to parenchymal tissue contamination. This creates confusion in the creatinine peak value, which is also the content in the neurocysticercosis cyst wall and needs to be confirmed further with tissue biopsy.

\section{CONCLUSION}

Magnetic resonance spectroscopy has a definitive complementary role in aiding the diagnosis of various intracranial cystic lesions.

\section{Summary}

108 patients with intracranial cysts were analysed in our study with in vivo proton multivoxel spectroscopy which provided additional metabolic information about them. Diagnosis was confirmed by histopathological examination and aspiration and culture in few cases. Lactate is found in most of the cysts, a non-specific finding. Abscesses can be further divided into aerobic, anaerobic and tuberculous by their specific peaks and treated with appropriate antibiotics. Hydatid and neurocysticercosis can be differentiated. Colloid cyst can be differentiated by presence of NAA peak from other benign cysts. High-grade tumours can be differentiated from low-grade tumours by presence of lipid and higher choline peak.

\section{Abbreviations}

MRS- Magnetic resonance spectroscopy, MRI- Magnetic resonance imaging, TE- Time to echo, TR- Time to repeat, Cho- Choline, Cr- Creatinine, NAA- N-Acetylaspartate.

\section{REFERENCES}

[1] Cousins JP. Clinical MR spectroscopy: fundamental, current applications and future potential. American Journal of Roentgenology 1995;164(6):1337-47.

[2] Gupta RK, Jobanputra KJ, Yadav A. MR spectroscopy in brain infections. Neuroimaging Clinics of North America 2013;23(3):475-98.

[3] Luthra G, Parihar A, Nath $K$, et al. Comparative evaluation of fungal, tubercular, and pyogenic brain abscesses with conventional and diffusion MR imaging and proton MR spectroscopy American Journal of Neuroradiology 2007;28(7):1332-8.

[4] Chang KH, Song IC, Kim SH, et al. In vivo single-voxel proton MR spectroscopy in intracranial cystic masses. AJNR: American Journal of Neuroradiology 1998;19(3):401-5.

[5] Poptani H, Gupta RK, Jain VK, et al. Cystic intracranial mass lesions: possible role of in vivo MR spectroscopy in its differential diagnosis. Magnetic Resonance Imaging 1995;13(7):1019-29.

[6] Lai PH, Li KT, Hsu SS, et al. Pyogenic Brain abscess: findings from in vivo 1.5-T and 11.7-T in vitro proton MR spectroscopy. Pan American Journal of Neuroradiology 2005;26(2):279-88.

[7] Garg M, Gupta RK, Husain M, et al. Brain abscesses: etiologic categorization with in vivo proton MR spectroscopy. RSNA Radiology 2004;230(2):519-27.

[8] Garg M, Chawla S, Prasad KN, et al. Differentiation of hydatid cyst from cyst cercus cyst by proton MR spectroscopy. NMR Biomedicine 2002;15(5):320-6.

[9] Lai PH, Ho JT, Chen WL, et al. Brain abscess and necrotic brain tumor: discrimination with proton MR spectroscopy and diffusion-weighted imaging. AJNR Am J Neuroradiology 2002;23(8):1369-77.

[10] Hingwala DR, Radhakrishnan N, Kesavadas C, et al. Neuroenteric cysts of the brain-comprehensive magnetic resonance imaging. Indian J Radiol Imaging 2013;23(2):155-63.

[11] Periakaruppan A, Kesavadas C, Radhakrishnan VV, et al. Unique MR spectroscopic finding in colloid-like cyst. Neuroradiology 2008;50(2):137-44. 